\title{
A Fully Automatic Calibration Framework for Navigated Ultrasound Imaging
}

\author{
Matthias Peterhans, Sylvain Anderegg, Philippe Gaillard, Thiago Oliveira-Santos, Stefan Weber
}

\begin{abstract}
Navigated ultrasound (US) imaging is used for the intra-operative acquisition of $3 \mathrm{D}$ image data during imageguided surgery. The presented approach includes the design of a compact and easy to use US calibration device and its integration into a software application for navigated liver surgery. User interaction during the calibration process is minimized through automatic detection of the calibration process followed by automatic image segmentation, calculation of the calibration transform and validation of the obtained result. This leads to a fast, interaction-free and fully automatic calibration procedure enabling intra-operative US calibration being performed by the surgical user.
\end{abstract}

\section{INTRODUCTION}

Ultrasound imaging is used for anatomical orientation and for intra-operative tumor detection during surgical interventions on the liver. Within our navigation system for liver surgery [1],[2] we use navigated ultrasound imaging to acquire information about organ deformation on the patient. Based on the 3D information about the location of liver vessels, registration methods for updating the pre-operative image data to the patient scenario are being developed [3].

The prerequisite for navigated ultrasound imaging is calibration of the ultrasound probe. Calibration establishes the relationship between a pixel in the ultrasound image and the corresponding location in 3D space by defining an image scaling transform and a 3D spatial transform relating the image pixels to the coordinate system of the marker shield which is attached to the ultrasound probe (Fig. 1).

As the navigation adapters are usually fixed onto the ultrasound probe during the surgical intervention, intraoperative probe calibration is strongly desirable. The requirements for an intra-operative calibration device is transportability and small size, compatibility with the sterile environment and a suitable calibration workflow which minimizes time requirements and enables calibration being performed by the surgical user.

A review [4] of existing ultrasound calibration solutions shows that most approaches proposed in literature are based on an object of known geometry placed in a water bath. Calibration is either performed by aligning the ultrasound probe to phantom features and acquiring one or several

All authors are Members of the ARTORG Center for Computer-Aided Surgery at the Institute for Surgical Technology and Biomechanics, University of Bern, Switzerland.

The Corresponding author is Matthias Peterhans, Email: matthias.peterhans@istb.unibe.ch , Tel +41 31631 5920, Address: ISTB, Stauffacherstrasse 78, CH-3014 Bern, Switzerland images (single-point [5], cross-wires [6], multiple point targets [7]) or by taking images of a specially designed phantom from arbitrary directions. From a clinical point of view, probe alignment to phantom features should be avoided in order to minimize complexity. Calibration time is minimized by using as few images as possible and by using automatic methods for detecting the required features in the ultrasound images. Furthermore, open water bath phantoms should be avoided because they are cumbersome to use in a sterile environment.

We present herein the development of a new calibration unit which fulfills the clinical requirements and enables intraoperative probe calibration to be done fully automatically and in a minimal amount of time.

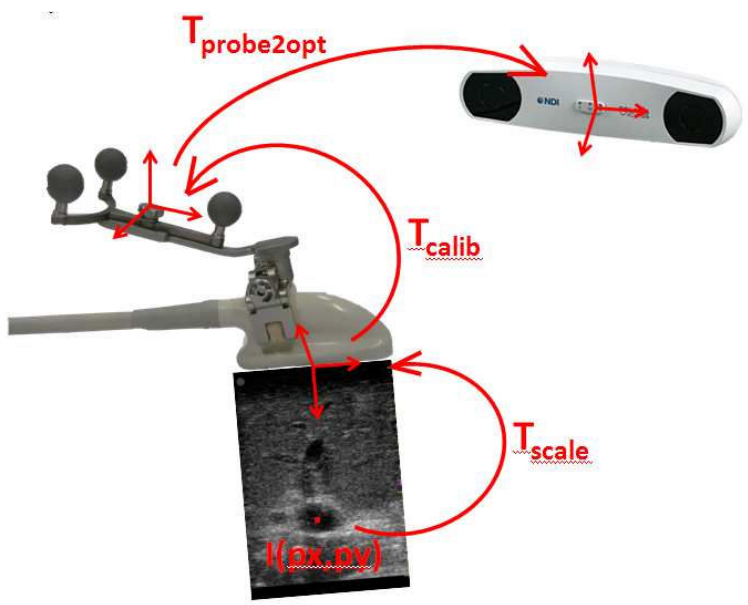

Fig. 1. Definition of the ultrasound calibration problem.

\section{METHODS}

\section{A. Navigation System and Ultrasound Device}

The ultrasound calibration framework is part of a surgical navigation system used in open liver surgery. The navigation system is built as a transportable setup containing an NDI Vicra Camera (Northern Digital Inc, Canada), an integrated Terason T3000 ultrasound system with an 8IOA intraoperative probe (Teratech Corporation, USA) and a Shuttle barebone PC (Shuttle Inc, Taiwan) with a touch-screen monitor (ELO Touchsystems, USA). Instrument tracking is enabled by a navigation toolset composed of adapters to the existing surgical tools (CUSA, ablation devices) and a pointer calibration unit. The toolset is complemented by the intra-operative US calibration unit described herein. 


\section{B. Calibration Hardware Design}

The calibration unit uses the principle of a Z-fiducial phantom [8]. This type of phantom was chosen because unlike other phantom types, only one image is required to conduct calibration and this image doesn't need to be aligned with a target [4]. Moreover, it presents the advantage that only one image from a predefined view direction is required. This enables the design of a closed shape with one opening for ultrasound imaging.

A good compromise between size of the calibration unit and precision was found using six Z-fiducials placed one above the other. In order to avoid the inherent indeterminacies of a Z-fiducial phantom (internal symmetries), one Z-fiducial was inversed and shortened.

The actual calibration phantom is enclosed by a waterfilled casing onto which a marker shield is screwed for use within the navigation system (Fig. 2). Through a direct connection between the phantom and the marker shield, precise determination of the wire locations in the marker coordinate system can be obtained from the construction data.

Image acquisition is performed through a silicone window on top of the calibration unit. For avoiding artifacts from air bubbles in the calibration unit, a degassing mechanism is implemented at the bottom of the calibration unit.

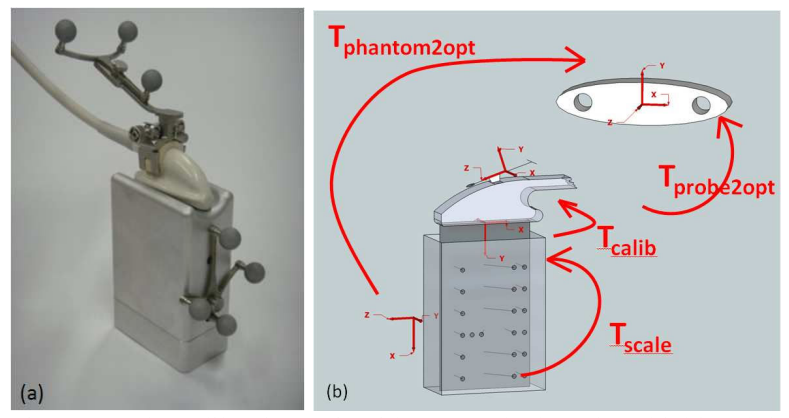

Fig. 2. a) The calibration unit with an attached marker shield and the navigated ultrasound probe. b) Coordinate conventions and transforms for the calibration process

\section{Automatic Wire Detection}

When imaging the Z-wire phantom, three collinear points appear for each Z-fiducial crossed by the US plane. This results to a total of 18 points on our phantom (with $6 \mathrm{Z}$-wires at different depth). In order to minimize the calibration time an automatic detection algorithm was developed to detect the phantom points.

The detection algorithm starts with local maxima detection in a 9-connected neighbourhood of each image pixel. The detected local maxima are then examined in decreasing intensity order to determine their respective connected regions. The criterion for a connected pixel with intensity $I\left(x_{c o n}, y_{c o n}\right)$ to lie within a region around the local maximum $I\left(x_{\max }, y_{\max }\right)$ is:

$I\left(x_{c o n}, y_{c o n}\right)>I\left(x_{\max }, y_{\max }\right)-T_{c o n}$ where $\mathrm{T}_{\text {con }}$ is the threshold for connected regions. All local maxima lying within a connected region of another local maximum with higher intensity are excluded from further processing. The parameter $\mathrm{T}_{\text {con }}$ is optimized for finding 50 points or less using a modified binary search algorithm. An image is rejected if $\mathrm{T}_{\text {con }}=\mathrm{I}\left(\mathrm{x}_{\text {may }}, \mathrm{y}_{\max }\right)$ leads to less than 50 points.

Once 50 points are detected, geometric criteria are applied for finding the 18 points corresponding to the desired phantom features. We know a priori that the 3 points of a Zfiducial are lying at the same depth and that the Z-fiducials are aligned vertically (except for the inverted fiducial) and distributed equally in depth. Based on this knowledge, points lying within a horizontal band of 3 pixels are grouped together and 6 groups of three points with regular depth spacing are selected. After that, the vertical alignment is tested by verifying that the points on the left and right lines lie within a band of 9 pixels. The central points are assumed to be correct if the angle between line segments defined by consecutive points is below 5 degree. If all the criteria are fulfilled and 18 image points $P_{\text {image }}$ remain, the calibration algorithm described in the next section is started.

\section{Calibration algorithm}

The 3D positions $P_{\text {phantom }}$ of the central intersection points on the phantom in each $\mathrm{z}$-wire is calculated using similar triangles and knowledge of the wires real positions [8].

The scaling factors $s_{x}$ and $s_{y}$ are assumed to be equal and are calculated from the average ratio between vertical distances between $P_{\text {phantom }}$ and the pixel separation between the corresponding $P_{\text {image }}$ :

$s_{x}=s_{y}=\frac{1}{n} \sum \frac{P_{\text {phantom }, i}(y)-P_{\text {phantom }, j}(y)}{P_{\text {image }, i}(y)-P_{\text {image }, j}(y)}$

Based on $T_{\text {scale }}$ the detected points from the wire segmentation algorithm are mapped from image pixels to the 3D image space using:

$$
P_{\text {image }, \text { scaled }}=T_{\text {scale }} P_{\text {image }}
$$

The phantom points $P_{\text {phantom }}$ are then mapped to the US probe coordinate space by:

$P_{\text {phantom }}^{\prime}=\operatorname{inv}\left(T_{\text {probe 2opt }}\right) T_{\text {phantom 2opt }} P_{\text {phantom }}$

where $T_{\text {probe2opt }}$ and $T_{\text {phantom2opt }}$ are $3 \mathrm{D}$ transform matrices respectively relating the US probe and phantom coordinate space to the camera coordinate space (Fig. 2b).

Finally, the calibration matrix $T_{\text {calib }}$ is calculated using Arun's pair-point matching algorithm [9] for solving:

$P_{\text {phantom }}^{\prime}=T_{\text {calib }} P_{\text {image , scaled }}$ 


\section{E. Workflow}

In order to minimize the required interaction, ultrasound probe placement on the calibration unit is detected by the liver navigation software. The application then switches automatically to the calibration mode where the ultrasound image of the phantom and the obtained segmentation are shown. When entering the calibration mode, imaging parameters are automatically set to optimal values for the ultrasound phantom.

As soon as 18 valid points are detected, calibration matrices $\mathrm{T}_{\text {scale }}$ and $\mathrm{T}_{\text {cal }}$ are calculated and a validation image is displayed. The user can then either select to repeat the calibration or leave the calibration mode by moving the ultrasound probe away from the calibration unit. Calibration can be repeated whenever required (changes in ultrasound settings, motion of the instrument adapter).

\section{F. Validation Methodology}

As the fundamental requirement for the ultrasound calibration framework was usability within the surgical workflow, the resulting time overhead was assessed by measuring the calibration times for a user performing 40 calibrations. Time measurement started when the system switched to the calibration mode and stopped once the calibration transform was calculated.

The repeatability of the calibration procedure is measured using the standard deviations of Euler angles and translation parameters obtained from 40 calibration experiments. For the accuracy validation, the theoretical intersection points between the Z-fiducial phantom and the US image plane are calculated based on the obtained calibration matrix and the tracking information. These are then compared to the detected US feature points which leads to a measure of accuracy in the determination of 3D locations from US image content.

\section{RESULTS}

\section{A. Image quality}

Fig. 3a shows an ultrasound image acquired on the imaging phantom. It can be seen that all the fiducials can be identified and only few US artifacts are visible on the image. The central wire intersections appear as elongated regions because of the oblique crossing of the wires with the US image plane. Optimal image quality was obtained using the high frequency setting of the Terason IO8A transducer and setting the time depth compensation (TDC) to equal values.

For obtaining realistic image acquisition conditions, a test with sterile covers around the calibration unit and the ultrasound probe was performed. These two additional layers of plastic between the US probe and the calibration unit did not affect the image quality.

\section{B. Automatic point detection and calibration}

A representative image showing the detected points (in green) can be seen in figure $3 \mathrm{~b}$. The criteria for the selection of the 18 points could be fulfilled in all the 40 calibration experiments after a probe alignment phase.

The average processing time for the point detection algorithm was $50 \mathrm{~ms}$.
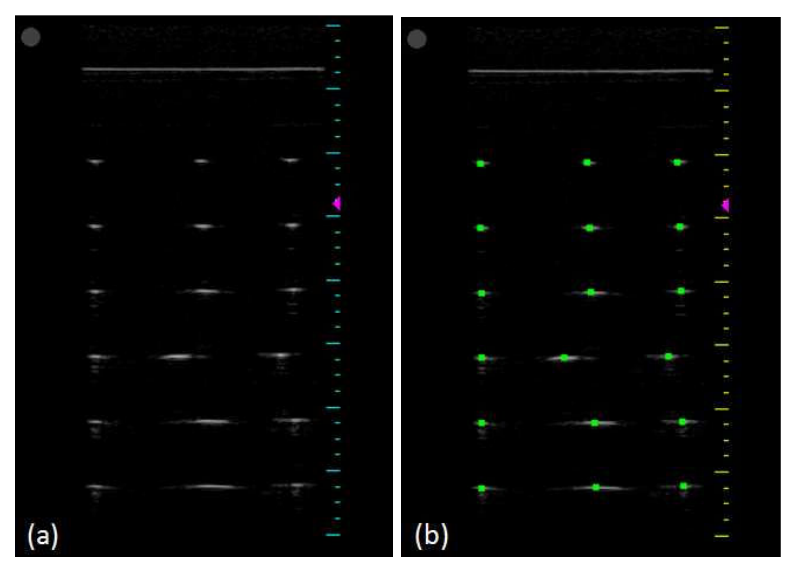

Fig. 3. a) Ultrasound image of the calibration phantom. b) Result of the automatic feature detection in green.

\section{Time requirements}

The median time required for the whole calibration procedure was 5 seconds with a range from 1 to 22 seconds. This includes probe alignment, automatic points detection, computation of the registration transform as well as the verification of the calibration through the calculation of the error on back-projected points.

\section{Calibration repeatability and accuracy}

The repeatability assessment of the calibration procedure is presented in table 1 by the mean values and standard deviations of the obtained Euler angles $(\alpha, \beta, \gamma)$, translation parameters $\left(t_{\mathrm{x}}, \mathrm{t}_{\mathrm{y}}, \mathrm{t}_{\mathrm{z}}\right)$ and the scaling factor $\mathrm{s}$.

TABLE I

REPEATABILITY OF THE CALIBRATION PROCEDURE

\begin{tabular}{lrr}
\hline \hline & Mean Values & Standard Deviation \\
\hline$\alpha$ [degree] & -62.8 & 0.4 \\
$\beta$ [degree] & 75.2 & 9.5 \\
$\gamma$ [degree] & -15.3 & 2.4 \\
$\mathrm{t}_{\mathrm{x}}[\mathrm{mm}]$ & -0.4 & 0.4 \\
$\mathrm{t}_{\mathrm{y}}[\mathrm{mm}]$ & 82.9 & 0.2 \\
$\mathrm{t}_{\mathrm{z}}[\mathrm{mm}]$ & -36.3 & 0.2 \\
$\mathrm{~s}[\mathrm{~mm} /$ pixel] & 0.19 & 0.00 \\
\hline \hline
\end{tabular}

\footnotetext{
Mean values and standard deviations of Euler angles $(\alpha, \beta, \gamma)$, translation parameters $\left(\mathrm{t}_{\mathrm{x}}, \mathrm{t}_{\mathrm{y}}, \mathrm{t}_{\mathrm{z}}\right)$ and the scaling factor $\mathrm{s}$.
}

Further evaluation in table 2 shows the mean errors according to the coordinate directions ( $\mathrm{x}$ and $\mathrm{y}$ in the image plane) obtained after back-projection of the wire intersections onto the US image.

\section{E. Workflow evaluation}

In all the calibration experiments, the automatic activation and deactivation of the calibration software worked correctly. Image segmentation always provided the required 18 points and launched the calibration process. 
TABLE II

BACK-PROJECTION ERRORS

\begin{tabular}{lrr}
\hline \hline & Mean Values & Standard Deviation \\
\hline $\mathrm{x}[\mathrm{mm}]$ & -0.01 & 0.29 \\
$\mathrm{y}[\mathrm{mm}]$ & 0.00 & 0.07 \\
$\mathrm{z}[\mathrm{mm}]$ & -0.09 & 0.21 \\
\hline \hline
\end{tabular}

\section{DISCUSSION}

We present a fully automatic and interaction-free calibration procedure for navigated ultrasound which is integrated into a framework for navigated liver surgery. The key components enabling automation of the calibration process are the well-known hardware setup and the seamless integration into the software concept. Through the direct interface to the ultrasound device, adequate imaging parameters can be predefined and lead to optimal image quality. Based on such images, fast and automatic wire detection is feasible and allows for calibration without user interaction. Through the automatic recognition of the probe placement on the calibration phantom in the navigation software, manual user interaction becomes obsolete.

The repeatability obtained with the current calculation algorithm is high for translations and two of the rotations parameters. The third rotation parameters shows a higher standard deviation which is due to the fact that all the detected phantom points lie close to a vertical line in the image. This makes the determination of the rotations around this line (y-coordinate in the image) difficult. Also, the precision of the phantom point detection decreases with the US image depth and the increased US beam thickness (see Fig. 3a). Adaptations in the wire placement could be used to improve this problem.

The resulting errors obtained by back-projecting the intersection points between the phantom and the computed US image plane are small. The high standard deviations of $\mathrm{x}$ and $\mathrm{z}$ are directly linked to the third rotation parameter mentioned above.

A further shortcoming of the current calibration approach is the use of a uniform scaling factor. This is correct as long as the speed of sound on the material being imaged corresponds to the assumed speed of the ultrasound device. For other tissues, a correction factor for the depth scaling $\mathrm{s}_{\mathrm{y}}$ is required. As the phantom uses a medium with known acoustic properties (water), the correction factor can be calculated based on the speed of sound in the tissue being imaged.

The ultrasound calibration unit is easy to handle (no water handling, small in size, no assembly required) and can be packed into a sterile bag. Fulfilling these basic OR requirements, ultrasound calibration can be performed during surgical interventions. Through the automation of the calibration process, the time overhead resulting from the preparation of navigated ultrasound imaging is reduced to a minimum ( $5 \mathrm{~s}$ median duration). This enables navigated ultrasound to be used as a routine imaging tool during liver surgery. In combination with the liver navigation system, ultrasound images are displayed as 3D textures within the anatomical context obtained from pre-operative images. This visualization provides better interpretability of the US images and can be used for guiding the US probe towards structures of interest (such as tumors, vessel bifurcations).

Our current research is using the obtained navigated ultrasound datasets for the development of US-based nonrigid registration algorithms to be used for updating the preoperative image data to the actual scenario on the patient.

\section{ACKNOWLEDGEMENTS}

We thank Urs Rohrer and Pascal Aebersold from the ISTB machine shop for valuable advice in the mechanical design process and the production of the calibration device.

\section{REFERENCES}

[1] M. Peterhans, B. Dagon, L. Nolte, C. Baur, A. Vom Berg, D. Inderbitzin, and S. Weber, "Soft tissue navigation: Transferring preoperative planning data into the operation room," ECR 2009 Book of Abstracts, Wien, 2009.

[2] A. Vom Berg, D. Candinas, D. Inderbitzin, M. Peterhans, S. Weber, and L. Nolte, "Computer assisted surgery and navigation in complex hepatic surgery and tumorablation: first clinical results of 10 patients with primary or secondary liver cancer," Accepted for the Proceedings of CARS 2010, Geneva: 2010.

[3] B. Dagon, C. Baur, and V. Bettschart, "Intraoperative update of liver surgery planning based on ultrasound images," Sauramps Medical, 2007, pp. 419-423.

[4] L. Mercier, T. Lang $\varnothing$, F. Lindseth, and D.L. Collins, "A review of calibration techniques for freehand 3-D ultrasound systems," Ultrasound in Medicine \& Biology, vol. 31, Apr. 2005, pp. 449-471.

[5] State A, Chen DT, Brandt A, et al, "Observing a volume rendered fetus within a pregnant patient, " Proceedings of IEEE Visualization, Los Alamitos, IEEE Computer Society Press, 1994, pp. $364-368$.

[6] Detmer PR, Bashein G, Hodges T, et al, "3D ultrasonic image feature localization based on magnetic scanhead tracking: in vitro calibration and validation," Ultrasound in medicine \& biology, vol. 20, 1994, pp. 923-936.

[7] J. Kowal, C.A. Amstutz, M. Caversaccio, and L.P. Nolte, "On the development and comparative evaluation of an ultrasound B-mode probe calibration method," Computer Aided Surgery, vol. 8, 2003, pp. 107-19.

[8] R.M. Comeau, A. Fenster, and T.M. Peters, "Integrated MR and ultrasound imaging for improved image guidance in neurosurgery," Medical Imaging 1998: Image Processing, K.M. Hanson, Ed., San Diego, CA, USA, SPIE, 1998, pp. 747-754.

[9] K.S. Arun, T.S. Huang, and S.D. Blostein, "Least-squares fitting of two 3-D point sets," IEEE Transactions on Pattern Analysis and Machine Intelligence, vol. 9, 1987, pp. 698-700. 